\title{
Morphometry and breast cancer II. Characterisation of breast cancer cells with high malignant potential in patients with spread to lymph nodes: preliminary
}

\section{results}

\author{
HC VAN DER LINDEN, ${ }^{*} \dagger$ JPA BAAK, $\dagger$ J LINDEMAN,* J HERMANS, \\ CJLM MEYER†
}

From the *Department of Pathology, Stichting Samenwerking Delftse Ziekenhuizen, Delft, the $\nmid$ Pathological Institute, Free University Hospital, Amsterdam, and the $\ddagger$ Department of Medical Statistics, State University, Leiden, The Netherlands

SUMMARY The prognostic value of clinical, quantitative, and qualitative microscopical features of both the primary tumour and also of the affected lymph nodes were investigated in 71 patients with breast cancer with spread to lymph nodes $\left(T_{x} N_{+} M_{0}\right)$. Age, tumour size, and localisation of the tumour comprised the clinical features; morphometry included assessment of the cellularity index, the mitotic activity index, and seven nuclear indices; the qualitative features investigated were histological type and grade, nuclear grade, oestrogen receptor content, number of lymph nodes affected, capsule infiltration of the nodes, presence of metastatic deposits in the efferent lymph vessels, percentage area of lymph node occupied by tumour. Immunohistochemistry was performed to show the presence of carcinoembryonic antigen and peanut agglutinin. All the patients had a mimum follow up of 24 months (maximum 48 months, mean 36 months).

Analysis of the results showed that the combined results of morphometry (of the primary tumour and the axillary lymph node metastatic deposits) yielded more information than analysis of axillary lymph node state, or morphometry of the primary tumour, or the lymph node metastases alone. Patients with a nuclear axes ratio of $>1.41$ in the primary tumour and $>1.36$ in the lymph node metastatic deposits were less likely to develop distant metastases than patients with values below any of these thresholds (recurrence rates $5 \cdot 2 \%$ and $46 \%$, respectively).

Thus the preliminary results of this prospective study indicate that morphometry provides important prognostic information in patients with breast cancer that has spread to lymph nodes.

The presence of axillary lymph node metastases is currently the most important criterion for treating patients with breast cancer with adjuvant therapy. ${ }^{12}$ Surgical dissection of axillary lymph nodes is a rather mutilating procedure, but because of the outstanding prognostic value of this criterion, attempts to replace it have so far failed. ${ }^{3-6}$ Several studies have shown that $30-45 \%$ of patients with lymph node metastases, who are not treated with any form of adjuvant therapy are free of disease after five years. ${ }^{78}$ A relatively high percentage of overtreatment is the result when the decision to treat these patients with adjuvant therapy is based solely on the presence of lymph node metastases. Identification of this "low risk" subset of Accepted for publication 18 December 1985 patients with spread to the lymph nodes would be an obvious advantage in patient care.

In a previous study, using nuclear morphometry of the primary tumour, we could not predict the presence or absence of lymph node metastases9: we showed that the primary tumour and the metastatic deposits in the lymph nodes are generally similar. It was also clear, however, that in the subgroup (about $25 \%$ of all patients) the nuclear shape differed in the metastases (either more ellipsoid or round) in spite of the similarity in size of the nuclei.

The aim of the present study was to investigate whether the morphometric findings in patients with breast cancer and spread to the lymph nodes had some prognostic value. Several quantitative and qual- 
itative microscopical features were compared to assess the possible independent or additional prognostic value of these features.

\section{Material and methods}

\section{PATIENTS}

Patients were included in this study, who had undergone surgery for primary breast cancer in one of the hospitals affiliated with the Stichting Samenwerking Delftse Ziekenhuizen (SSDZ), Delft, The Netherlands, between January 11981 and December 31 1982, who had no other overt disease, had adequate follow up, and had more than eight investigated axillary lymph nodes. All tumours were carefully reviewed by two of us (HCL and JPAB). Only patients with an adenocarcinoma of the "not otherwise specified" (NOS) ductal type ${ }^{10}$ with spread to the lymph nodes $(n=77)$, were used for further analysis. ${ }^{9}$ During follow up six patients were excluded from the study because the follow up was inadequate (due to death from causes other than recurrence, or moving to another city within twelve months after the operation). The mean age was 58.4 years (median 58 , range 26-88), and the mean tumour size $3 \cdot 1 \mathrm{~cm}$ (median 2.5, range 0.9-10): the material was very similar to that of other studies. ${ }^{711}$ The minimum follow up was 24 months (maximum 48, mean 36).

All patients were examined every three months during the first two years after surgery and every six months thereafter. In accordance with WHO recommendations, ${ }^{12}$ the fact that disease had recurred (reappearance of old or appearance of new lesions) was accepted if microscopically confirmed, or if a combination of clinical, x-ray, scintigraphical and biochemical data were conclusive. Only distant metastases were regarded as "recurrence". During the entire follow up period 45 patients had no recurrence $(63 \%)$.

\section{MORPHOMETRIC METHODS}

Details of morphometry and theoretical background have been described elsewhere ${ }^{13-16}$ : earlier publications outline the application to breast cancer. ${ }^{17141839}$ Briefly, morphometry was performed on standard $4 \mu \mathrm{m}$ thick, haematoxylin and eosin stained paraffin sections, including the centre and the periphery of the tumour. The material was fixed for at least 24 hours in $4 \%$ buffered formalin. Material that had been used for frozen section procedure was excluded because of collapse of the nuclei.

The following features were assessed in the most cellular areas of the tumour (usually at the periphery): The mitotic activity index This was defined as the number of mitoses in 10 high power fields (magnification $\times 400 ; \times 40$ objective numerical aperture 0.75 , field diameter at specimen leveP $450 \mu \mathrm{m})$. The counting was started in the most activeo area of the tumour (where the mitotic activity was highest). The subsequent nine contiguous fields weres selected at random, and no attempts were made tog maximise the counting by selecting areas with a high를 number of mitoses. Only definite mitotic figures weres counted, using the following criteria to distinguish mitotic figure from a pyknotic deformed nucleus absence of nuclear membrane; absence of a clear zone in centre; presence of "hairy" projections; and baso-음 philic cytoplasm.

The cellularity index This was the mean of the num ber of nuclei counted in five fields at $\times 1000 \overline{8}$ magnification. One field superimposed on thei projection screen is divided by a grid of lines into 36 square of $16 \mu \mathrm{m}^{2}$ each at specimen level. Only epios thelial cell nuclei not in contact with any of the mare gins and with an average diameter $>4 \mu \mathrm{m}$ at speci-윽 men level were counted.

The nuclear measurements These were performed at $\times 1000$ magnification on a commerically available graphic tablet (Mop-Videoplan, Kontron, Munich $\overrightarrow{0}$ West Germany) using microphotographs. Theू nuclear features assessed were perimeter, area, short:est axis, longest axis, diameter, shape factor $(4 \pi \times 0$ area/perimeter ${ }^{2}$ ), and axes ratio. Twenty five nuclei of tumour cells were measured, and the mean and stan dard deviation (SD) of each feature were calculate for each specimen. In total 14 nuclear variables were assessed. The size independent shape factor was $\vec{\delta}$ measure of the roundness and regularity of the profile, being 1.0 in perfectly regular, round nucle and $<1.0$ in other structures.

Morphometry was performed on both the primary tumour and in the lymph node metastatic deposits. A? morphometry of lymph node metastases is not ofter performed several control studies were carried out to examine the quality of the measurements, as described in an earlier publication. Morphometry was a highly reproducible method, both on the primary tumour and in the lymph node metastases. ${ }^{9}$

\section{QUALITATIVE FEATURES STUDIED}

In the lymph nodes capsule infiltration and efferent lymph vessel invasion were graded as absent (I) oर present (II). Fisher et al give a detailed description of these assessments. ${ }^{19}$ The fractional area of lymple nodes occupied by metastatic tumour deposits wa computed as the percentage of the whole section area In cases with more than one positive lymph node the mean percentage area of all the affected lymph nodes was taken for further analysis. The absolute numbe ${ }^{\circ}$ of positive lymph nodes was also documented.

Nuclear grading of the sections was performed according to the method of Black et al, ${ }^{21}{ }^{21}$ in whict 
the grading of the structures was classified from I to III. Grade III represents the group with good differentiation, grade II moderate differentiation, and grade I poor differentiation. Histological grading was performed according to the method of Bloom and Richardson. ${ }^{22}$ Using this method, tumours are also graded from I to III, but here, histological grade I represents tumours with a good differentiation and grade III those with poor differentiation.

Carcinoembryonic antigen and peanut lectin were assessed by using an indirect immunoperoxidase method, as described previously. ${ }^{23}$ These tumour markers were graded from 0 to 4 , representing an increasing presence of the investigated marker.

The oestrogen receptor content was determined with a biochemical assay, in which values above $11 \mathrm{fmol} / \mathrm{g}$ bound oestradiol were classified as positive (2), if less then $9 \mathrm{fmol} / \mathrm{g}$ interpreted as negative (1), and figures in between as doubtful. ${ }^{8}$

\section{STATISTICS}

The main interest of this study was to investigate whether the appearance of recurrence-that is, distant metastases-could be predicted by morphometry. This problem was tackled by survival analysis as well as by comparison of the group with recurrence with the group free of recurrence. To avoid bias introduced by varying lengths of follow up in the group with recurrence 24 months of follow up was taken as a fixed end point. The actual state of the patients at 24 months was used to define the two groups. At that time 50 patients were free of recurrence and 21 had developed a distant metastasis. The same analysis was performed for all the other investigated (qualitative and quantitative) features. The comparison of the two groups was carried out using the Fisher test, Wilcoxon's rank sum test, and the $\chi^{2}$ test.

To investigate the simultaneous prognostic value of primary tumour and lymph node nuclear morphometry several combinations of different values of the nuclear morphometric features, as decision thresholds, were evaluated using the $\chi^{2}$ test.

Actuarial curves for recurrence were calculated by the Kaplan-Meier method. For this analysis the fixed end point was abandoned and the maximum available follow up for each patient was used. Five more patients had recurrence after 24 months; overall, 45 patients were free of recurrence and 26 developed a distant metastasis. Statistical differences were determined by the Mantel-Cox test using the program P1L from the BMDP package.

For all analyses probabilities of $<0.05$ were regarded as significant.

\section{Results}

The Table summarises the mean values of the morphometric, all other investigated (quantitative, qualitative, and oestrogen receptor) features, and the statistical difference between the groups with and without recurrence (with a fixed follow up of 24 months). With single variate analysis, only the number of positive nodes, the percentage area of the lymph node occupied by tumour, the oestrogen receptor state of the primary tumour, the mean mitotic activity index, the standard deviation of the nuclear shape factor, and the mean and standard deviation of

Mean values and statistical analysis of qualitative and quantitative features that were investigated

\begin{tabular}{|c|c|c|c|c|c|c|}
\hline & \multicolumn{2}{|c|}{ No recurrence $(n=50)$} & \multicolumn{2}{|c|}{ Recurrence $(n=21)$} & \multicolumn{2}{|c|}{$\begin{array}{l}\text { Comparison of groups with and } \\
\text { without recurrence* }\end{array}$} \\
\hline & $\begin{array}{l}\text { Primary } \\
\text { tumour }\end{array}$ & $\begin{array}{l}\text { Lymph } \\
\text { node }\end{array}$ & $\begin{array}{l}\text { Primary } \\
\text { tumour }\end{array}$ & $\begin{array}{l}\text { Lymph } \\
\text { node }\end{array}$ & $\begin{array}{l}\text { Primary } \\
\text { tumour }\end{array}$ & $\begin{array}{l}\text { Lymph } \\
\text { node }\end{array}$ \\
\hline $\begin{array}{l}\text { Mean No of positive lymph nodes } \\
\text { Capsule infiltration }(\text { absent }=1 \text {, present }=2 \text { ) } \\
\text { Efferent lymph vessel invasion }(\text { absent }=1 \text {, present }=2 \text { ) } \\
\text { Areal lymph node percentage occupied by tumour } \\
\text { Oestrogen receptor state (negative }=2 \text {, positive }=1 \text { ) } \\
\text { Nuclear grade (I-III) } \\
\text { Histological grade (I-III) } \\
\text { Carcinoembryonic antigen }(0-4) \\
\text { Peanut lectin }(0-4)\end{array}$ & $\begin{array}{l}1.2 \\
1.6 \\
2.2 \\
2.0 \\
1.8\end{array}$ & $\begin{array}{l}4.0 \\
1.7 \\
1.8 \\
70 \\
1.7 \\
2.1 \\
1.9 \\
1.9\end{array}$ & $\begin{array}{l}1.7 \\
1.5 \\
2.4 \\
1.9 \\
1.6\end{array}$ & $\begin{array}{r}9.0 \\
1.5 \\
2.0 \\
83 \\
1.3 \\
2.3 \\
1.7 \\
1.8\end{array}$ & $\begin{array}{l}<0.01 \\
\text { ns } \\
\text { ns } \\
\text { ns } \\
\text { ns }\end{array}$ & $\begin{array}{l}<0.005 \\
\text { ns } \\
\text { ns } \\
<0.05 \\
\text { ns } \\
\text { ns } \\
\text { ns } \\
\text { ns }\end{array}$ \\
\hline $\begin{array}{l}\text { Morphometric features }{ }^{* *} \\
\text { Mean mitotic activity index } \\
\text { Mean cellularity index } \\
\text { Nuclear perimeter mean (SD) } \\
\text { Nuclear area } \\
\text { Shortest nuclear axis } \\
\text { Longest nuclear axis } \\
\text { Nuclear diameter } \\
\text { Nuclear shape factor } \\
\text { Nuclear axes ratio }\end{array}$ & $\begin{array}{l}11.0 \\
10 \cdot 7 \\
25 \cdot 1(3 \cdot 1) \\
43 \cdot 8(10 \cdot 8) \\
6 \cdot 4(0 \cdot 95) \\
8 \cdot 8(1 \cdot 32) \\
7 \cdot 3(0 \cdot 89) \\
0.85(0 \cdot 05) \\
1 \cdot 39(0 \cdot 24)\end{array}$ & $\begin{array}{l}11 \cdot 3 \\
8 \cdot 5 \\
24 \cdot 7(2 \cdot 8) \\
45 \cdot 0(10 \cdot 2) \\
6.4(0 \cdot 92) \\
9.0(1 \cdot 25) \\
7.5(0 \cdot 84) \\
0.90(0 \cdot 06) \\
1.44(0 \cdot 27)\end{array}$ & $\begin{array}{l}15.5 \\
9 \cdot 3 \\
26 \cdot 5(3 \cdot 3) \\
49 \cdot 3(13 \cdot 1) \\
6 \cdot 5(1 \cdot 02) \\
9 \cdot 2(1 \cdot 35) \\
7.8(0 \cdot 99) \\
0.85(0 \cdot 05) \\
1 \cdot 36(0 \cdot 22)\end{array}$ & $\begin{array}{l}18.9 \\
8.0 \\
25.2(2.6) \\
47.1(10.0) \\
6.7(0.90) \\
9.0(1.11) \\
7.6(0.80) \\
0.91(0.05) \\
1.38(0.22)\end{array}$ & $\begin{array}{l}\text { ns } \\
\text { ns } \\
\text { ns } \\
\text { ns } \\
\text { ns } \\
\text { ns } \\
\text { ns } \\
\text { ns } \\
\text { ns }\end{array}$ & $\begin{array}{l}<0.05 \\
\text { ns } \\
\text { ns } \\
\text { ns } \\
\text { ns } \\
\text { ns } \\
<0.01 \\
<0.05 \\
<0.01\end{array}$ \\
\hline
\end{tabular}

*Fisher test, Wilcoxon's test, $\chi^{2}$ test; $n$ s = not significant $(\mathrm{p}>0.05)$.

** SD = Standard Deviation. 


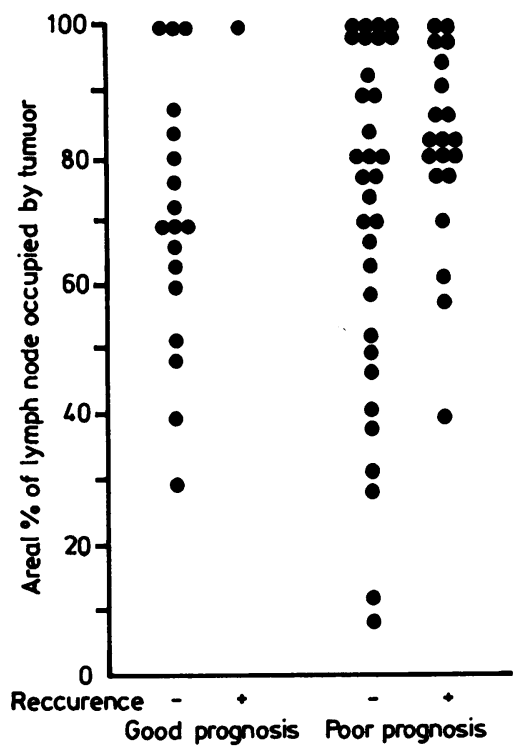

Fig. 1 Percentage of area of lymph node occupied by tumour in patients predicted by combined nuclear axes ratio measurement to develop recurrence correlated to actual number with recurrence. Note considerable overlap in area percentage in spite of tendency towards higher values in patients with recurrence. Good prognosis and poor prognosis refer to two groups of patients discerned on the bases of their mean nuclear axes ratio, as visualised in Fig. 2.

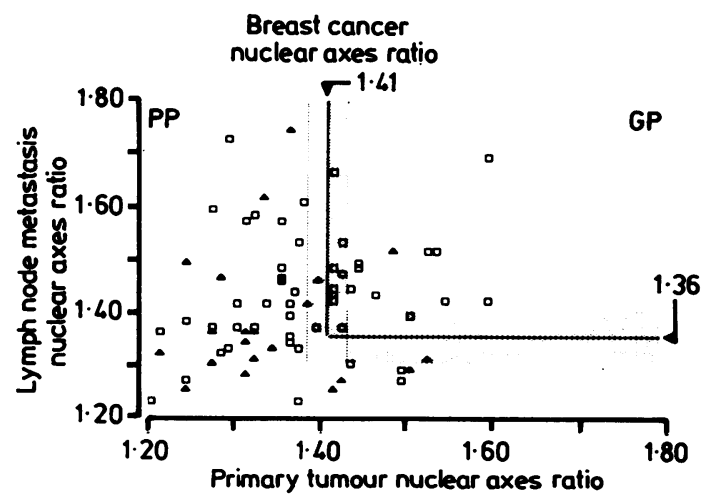

Fig. 2 Comparison between mean nuclear axes ratio of primary tumour and lymph node metastases of patients with breast cancer with and without recurrence.

Note in group with good prognosis (GP) one patient showed recurrence of her tumour, and 18 patients were free of recurrence. In group with poor prognosis (PP) 20 patients had recurrence; 32 did not. Group with good prognosis contains significantly more patients without recurrence $\left(\chi^{2}=7.3 v=1 ; p<0.05\right)$. After changing vertical threshold between 1.37 and 1.45 , or horizontal threshold between 1.32 and 1.40 (striated area) classification result was still significant.

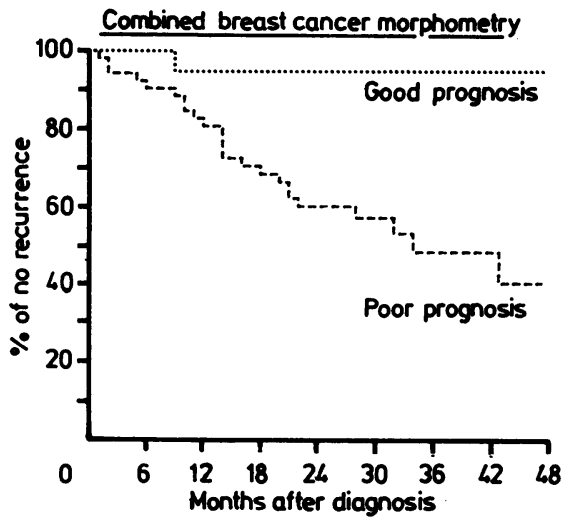

Fig. 3 Kaplan-Meier survival curve of patients with good prognosis and poor prognosis who had spread to lymph nodes, identified by combined nuclear breast cancer morphometry asco defined in Fig. 2. (Matel-Cox; $9.05 p=0.026$.)

the nuclear axes ratio were significantly correlated with recurrence. In patients with breast cancer, buF without recurrence the tumours are more oftenoestrogen receptor positive, there are less positiveso lymph nodes, and the metastases occupy a smaller percentage area of the lymph nodes - with a consido erable overlap (Fig. 1).

Multiple linear regression selected the combined mean nuclear axes ratio of the primary tumour anof the lymph node metastatic deposit and the percentage area of the lymph node occupied by tumour as the best combination with the highest correlation with of without recurrence $\left(\mathrm{r}^{2}=0.25 ; \mathrm{p}<0.005\right)$. No important information was deduced from the other features investigated.

Percentage area measurements added very little to combined nuclear axes ratio morphometry, and spe $\frac{}{3}$ cial attention, therefore, was paid to the two means of the nuclear axes ratio. The bivariate nuclear axies? ratio graph (Fig. 2) showed that a high concentration of the patients free of recurrence were found in the right upper corner (with ellipsoid nuclei both in the primary tumour and in the lymph node metastatic. deposits). To determine optimal boundaries to dis $N$ tinguish good prognosis and poor prognosis sub groups the $\chi^{2}$ minimalisation technique was used to evaluate different thresholds of the nuclear axes ratio ${ }^{\omega}$ with 0.02 steps. In total, 48 combinations of differen? decision thresholds were evaluated. Using values of the nuclear axes ratio above 1.41 in the primary tumour and above 1.36 in the lymph node metastases as cut off points, only one patient of $19(5 \cdot 2 \%)$ devel $\frac{0}{-}$ oped metastatic disease (Fig. 2). Of the remaining patients, 20 of 52 had a recurrence during the follow? up period $(p<0.05)$. A recurrence free survival curve (Kaplan-Meier) of low and high risk patients (Fig. 3) 
confirms the prognostic difference between these two groups (Mantel-Cox: 9.04, $\mathrm{p}=0.0026$ ).

The reproducibility of the assessments of mean nuclear axes ratio was extensively tested in blind duplicate assessments by different investigators ${ }^{9}$ and was found to be satisfactory ( $r>0.90, p>0.005)$. The intraobserver and interobserver measuring differences of the axes ratio in the duplicate assessments performed did not exceed 0.03 . When changing the vertical threshold with a value of 0.04 between 1.37 and 1.45 , or the horizontal threshold between 1.32 and 1.40 , the classification results remained significant $(p<0.05)$. Fig. 2 shows the optimum best discrimination which could be obtained and it seems that this method of classification is worthwhile.

\section{Discussion}

Extensive evaluation of several features, including combined morphometry of the primary tumour and the lymph node metastatic deposits, was performed in 71 patients with primary breast cancer. After 24 months of follow up, 21 patients had recurrent disease, figures which agree with those of other studies. ${ }^{11}$ We found that, although some of the qualitative features were prognostically important, nuclear shape, morphometry of the primary tumour, and lymph node metastatic deposits was a strong predictor of the likelihood of recurrence. Therefore, the two nuclear shape features - that is, nuclear axes ratio and nuclear shape factor-were investigated more intensively, and bivariate plots of these features in the primary tumour and the lymph node metastatic deposits were computed in relation with recurrence, or lack of it. Using the $\chi^{2}$ test, different values of these features, when used as decision thresholds for recurrence, were tested for significance. For the mean nuclear shape factor the level of significance was not reached

$(p=0.08)$. It was clear, however, that the degree of ellipsoidity of the nuclei, when assessed both in the primary tumour and in the lymph node metastatic deposits, had the strongest prognostic value.

Taking the value of 1.41 and 1.36 of the mean nuclear axes ratios in the primary tumour and lymph nodes, respectively, as the decision thresholds, two groups of patients were discerned (Fig. 2). In the group with good prognosis (with a high axes ratio or, in other words, elongated ellipsoid nuclei) only one of 19 patients $(5 \cdot 2 \%)$ developed recurrence in the follow up period, whereas in the group with poor prognosis the rate of recurrence was much higher (roughly $50 \%$ ).

The only patients with a good prognosis who had a recurrence was oestrogen receptor positive, and none of the oestrogen receptor negative patients has had a recurrence at the time of writing. Of patients with poor prognosis who had a recurrence, more than $50 \%$ were oestrogen receptor positive. The prognostic information offered by the number of positive nodes and the oestrogen receptor state seems to have been completely eclipsed by the combined nuclear axes ratio.

It is striking that the percentage of patients without recurrence in the group was higher than $90 \%$. Furthermore, 19 patients with good prognosis out of a total of $71(27 \%)$ is similar to the expected number with spread to the lymph nodes who remain disease free in long term studies $(25-40 \%) .{ }^{711}$ It should be emphasised that these tentative results must be interpreted cautiously because of the relatively short follow up. The question that remains to be answered is whether with a longer follow up the patients with recurrence would again mainly be found in the "nonellipsoid nuclei" group with poor prognosis (Fig. 2).

Several of the patients in this group showed incongruity of nuclear shape (upper left and lower right corner of Fig. 2-that is, cells were more or less round in the lymph node metastatic deposits than in the primary tumour). This may have been caused by sampling errors of the analysed areas. As described in detail in a previous paper, however, this is not a very likely explanation for the discrepancy, as extensive recutting, remeasurement, and measurement of different lymph node metastatic deposits gave the same results. ${ }^{9}$ Dissemination of different clones of tumour cells probably has a more important role and requires further attention. The rates of recurrence in those patients with incongruities in their nuclear shapes were the same as those for patients in the left lower corner of Fig. 2 (round nuclei both in the primary tumour and lymph node metastases; $46 \%$ ), and therefore the tumours of these patients should be regarded as more malignant than those located in the group with good prognosis. The presence of rounder nuclei (either in the primary tumour or in the lymph node metastatic deposits) may indicate a more malignant behaviour pattern.

Further characterisation of these cells with high malignant potential is desirable. Although the tumours of patients with recurrence were more often oestrogen receptor negative (as was expected) and the axillary tumour load was somewhat higher (as reflected in the number of positive lymph nodes and the percentage area of the lymph nodes occupied by tumour), these features were often interchangeable in the low and high risk groups. Therefore, these features cannot accurately characterise the highly malignant breast cancer cells in these patients with spread to the lymph nodes. Unfortunately, the single patient in the low risk group with recurrence was oestrogen receptor positive, and none of the immuno- 


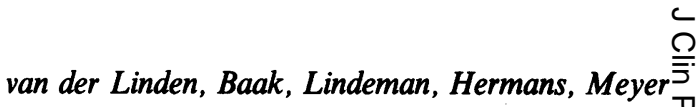

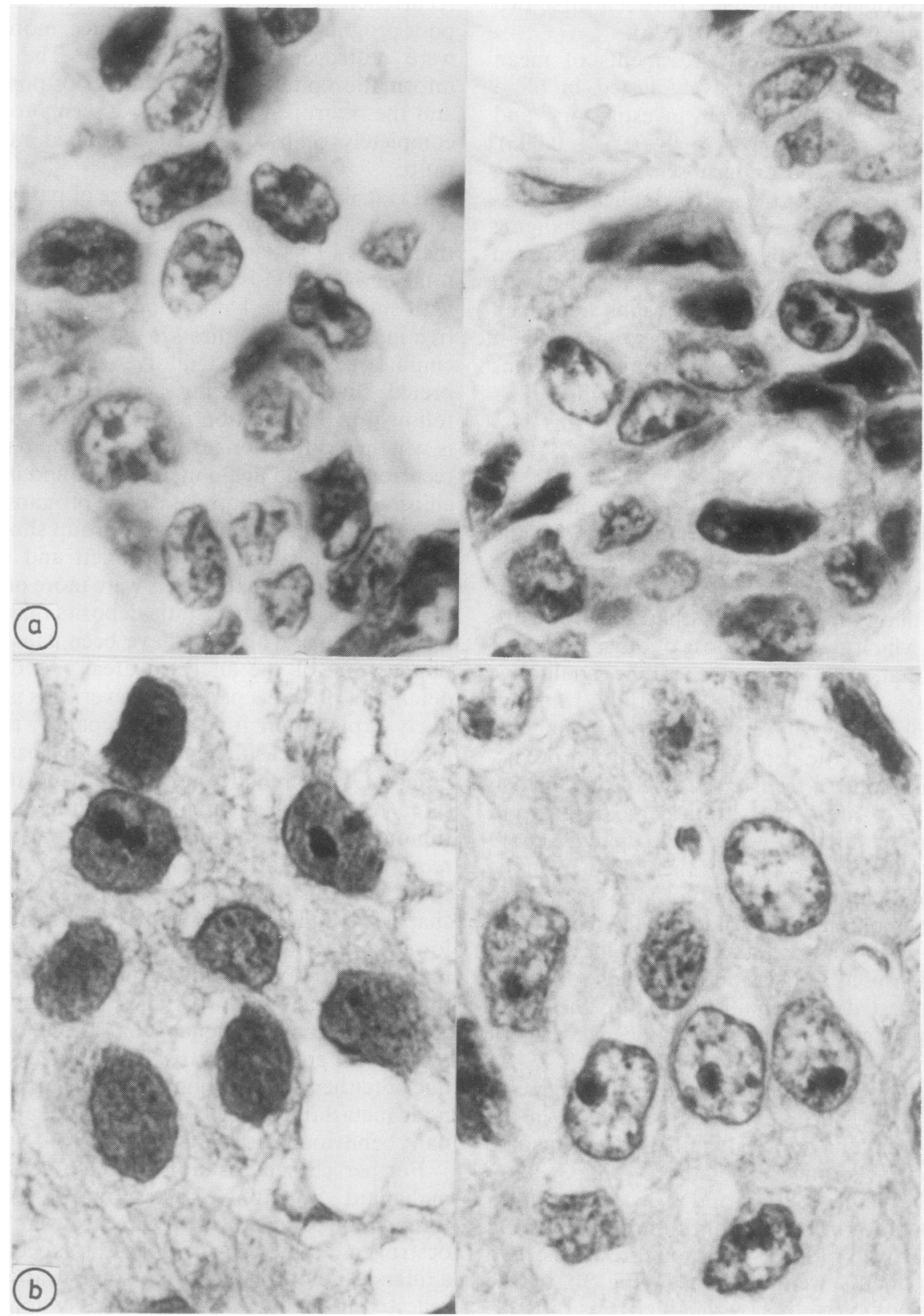

Fig. 4a Primary tumour (left) and lymph node metastasis (right) of patient without recurrence 40 months after operation. Note slightly irregular and elongated cells. Nuclear axes ratio in primary tumour 1.45 and in lymph node metastases 1.52. (Haematoxylin and eosin.) $\times 1300$.

Fig. 4b Primary tumour (left) and lymph node metastasis (right) of patient with recurrence. This patient died from breast cancer 2.5 years after operation. Nuclear axes ratio in primary tumour is 1.31 and in lymph node metastases 1.22. Note somewhat larger nuclei than those of Fig. 4 a.

Chromatin pattern and nuclear size are not clearly different. (Haematoxylin and eosin.) $\times 1300$. 
histopathological features investigated provided any additional prognostic information. Perhaps purification with cell sorting will be of help, as Tosi et al, ${ }^{24}$ and Maehle $e$ al $^{25}$ found larger mean nuclear areas in the lymph node metastatic deposits than in the primary tumours.

The nuclear and histological grade, morphometric features, and the qualitative and the immunohistochemical features were only mildly or not prognostically important, and none of the other qualitative features studied (including tumour size, age, and localisation of the tumour) predicted the outcome as well as did combined nuclear ellipsoid morphometry.

Thus tumours with nuclei that are rounder are more prone to recur. Fig. 4a shows the histological picture of a primary tumour and its lymph node metastatic deposit in a patient without recurrence (after 42 months follow up). Fig. 4b shows a histological picture of a ductal breast cancer from a patient with recurrence eight months after operation. It must be emphasised that the tumours illustrated are two extremes of a continuous spectrum of morphological appearances. Other tumours with less distinctive nuclear appearances were not classifiable with more subjective qualitative evaluation. Blind evaluation of all the slides showed that neither subjective assessment of the degree of ellipsoidity, nor nuclear or histological grade could give results comparable with those of combined morphometry.

Despite the reservations inherent in a preliminary study our findings may help other investigators to further characterise breast cancer cells with a higher malignant potential. The follow up is still being updated, so that more definite conclusions can be presented in the future.

This work was supported by grant No 28-735 from the Praeventiefonds.

\section{References}

${ }^{1}$ Fisher ER, Sass R, Fisher B. Pathologic findings from the national surgical adjuvant project for breast cancers (protocol No 4). Cancer 1984;53:712-23.

${ }^{2}$ Henderson IC, Canellos GP. Cancer of the breast. The past decade. $N$ Engl J Med 1980;103:17-20, 78-90.

${ }^{3}$ Baak JPA, Dop van H, Kurver PHJ, Hermans J. The value of morphometry to classical prognosticators in breast cancer. Cancer 1985;86:374-82.

${ }^{4}$ Sharkey FE, Pavlak RJ, Greizer AS. Morphometric analysis of differentiation in human breast carcinoma. Arch Pathol Lab Med 1983;107:406-10.

${ }^{5}$ Stenkvist B, Bengtson E, Dahlqvist B, et al. Predicting breast can- cer recurrence. Cancer 1982;50:2884-93.

${ }^{6}$ Zajdela A, Saravia dela Riva L, Ghossein NA. The relation of prognosis to the nuclear diameter of breast cancer cells obtained by cytologic aspiration. Acta Cytol 1979;23:75-80.

${ }^{7}$ Lythgoe JP, Leck I, Swindell R. Manchester regional breast study: preliminary results. Lancet $1978 ;$;:744-77.

${ }^{8}$ EORTC Breast Cancer Cooperative Group. Revision for the assessment of hormone receptors in human breast cancer. Report of the second EORTC workshop. European Journal of Cancer 1980;16:1513-15.

${ }^{9}$ Linden van der HC, Baak JPA, Smeulders AWM, Lindeman J. Meijer CJLM. Morphometry of breast cancer. I. Comparison of the primary tumours and the axillary lymph node metastases. Pathol Res Pract (in press).

${ }^{10}$ Azzopardi JG, Chepick OF, Hartmann WH, etal. The WHO histological typing of breast tumours. Am J Clin Pathol 1982;78:806-16.

${ }^{11}$ Nemoto K, Vana J, Bedwani RN, Baker HW, McGregor FH, Murphy GP. Management and survival of female breast cancer: results of a national survey by the American college of surgeons. Cancer 1980;45:2917-24.

12 WHO. Handbook for reporting results of cancer treatment. Geneva: World Health Organisation 1979:28-30.

${ }^{13}$ Aherne WA, Dunnill MS. Morphometry. London: Edward Arnold, 1982.

${ }^{14}$ Baak JPA, Oort J. A manual of morphometry in diagnostic pathology. New York: Springer-Verlag, 1983.

${ }^{15}$ Oberholzer M. Morphometrie in der klinischen pathologie. Heidelberg: Springer Verlag, 1983.

${ }^{16}$ Weibel ER, Elias H. Quantitative methods in morphometry. Berlin: Springer-Verlag, 1967.

${ }^{17}$ Baak JPA, Snoo-Nieuwlaat de AJE, Graef de S, Makkink B, Boon M. Prognostic indicators in breast cancer-morphometric methods. Histopathology 1982;6:327-39.

${ }^{18}$ Baak JPA, Persijn JP. In search of the best qualitative microscopical or morphometrical predictor of estrogen receptor in breast cancer. Pathol Res Pract 1984;187:307-14.

${ }^{19}$ Fisher ER, Gregorio RM, Fisher B. The pathology of invasive breast cancer. Cancer 1975;36:1-85.

${ }^{20}$ Black MM, Speer FD. Nuclear structure in cancer tissues. Journal of Surgical Gynecology and Obstetrics 1957;105:97-102.

${ }^{21}$ Black MM, Barclay THC, Hankley BF. Prognosis in breast cancer utilizing histologic characteristics of the primary tumor. Cancer 1975;36:2048-55.

${ }^{22}$ Bloom HJG, Richardson WW. Histological grading and prognosis in breast cancer. Br J Cancer 1957;11:359-77.

${ }^{23}$ Linden van der HC, Baak JPA, Smeulders AWM, Lindeman J. Meijer CJLM. Carcinoembyronic antigen (CEA) expression and peanut agglutinin (PNA) binding in primary breast cancer and lymph node metastases. Lack of correlation with clinical, histopathological, biochemical and morphometric features. Histopathology 1985;9:1051-9.

${ }^{24}$ Tosi P, Luzi P, Sforza V, Spina D, Miracco C, Santopietro R. Morphometrical assessment of mean nuclear area in breast cancer in comparison with that of lymph node metastases. Pathol Res Pract (in press).

${ }^{25}$ Maehle BO, Thorresen S, Skjaerven R, Hartveit F. Mean nuclear area and histological grade of axillary node tumour in breast cancer, related to prognosis. Br J Cancer 1982;46:95-100.

Requests for reprints to: Dr JPA Baak, Pathological Institute, Free University Hospital de Boelelaan 1117, 1007 MB Amsterdam, The Netherlands. 